\title{
Expression of CD44 on bile ducts in primary sclerosing cholangitis and primary biliary cirrhosis
}

\author{
S M Cruickshank, J Southgate, J I Wyatt, P J Selby, L K Trejdosiewicz
}

\begin{abstract}
Aim-To examine expression of CD44, a transmembrane glycoprotein involved in lymphocyte homing and activation, in inflammatory liver diseases.

Methods-Formalin fixed, paraffin embedded tissues were obtained from normal, uninvolved liver from patients undergoing partial hepatectomy for metastatic carcinoma (9) and transplant hepatectomy specimens from patients with primary biliary cirrhosis (12), primary sclerosing cholangitis (8), autoimmune hepatitis (3), hepatitis C (3), and secondary sclerosing cholangitis (1). Expression of CD44 (using antibodies to three core epitopes), HLA-DR, and lymphocyte phenotypic markers was studied by immunohistochemistry.

Results-CD44 expression was not detected in either hepatocytes or biliary epithelial cells in normal livers. In sections from all 27 transplant hepatectomy specimens, CD44 was positive in bile duct epithelial cells but not in hepatocytes. The proportion of $\mathrm{CD}_{4} 4^{+}$ducts was much higher in biliary disease than in chronic hepatitis. By contrast, expression of HLA-DR was detected in a relatively small percentage of bile ducts. Activated, memory phenotype $\mathrm{CD}^{+} \mathrm{T}$ lymphocytes were increased in the parenchyma of all diseased livers and an infiltrate of activated $\mathrm{CD8}^{+}$cells within the biliary epithelium was evident in inflammatory biliary disease.

Conclusions-CD44 appears to play an important role in the development of autoimmune biliary disease by promoting lymphoepithelial interactions, whereas HLA-DR may be involved in the subsequent progression of these conditions.

(f Clin Pathol 1999;52:730-734)
\end{abstract}

Research Unit, St James's University

Hospital, Leeds

LS9 7TF, UK

S M Cruickshank

J Southgate

P J Selby

L K Trejdosiewicz

Department of Pathology, St James's University Hospital J I Wyatt

Correspondence to: Dr Trejdosiewicz.

email:

1.k.trejdosiewicz@leeds.ac.uk

Accepted for publication 17 June 1999 Keywords: CD44; biliary epithelium; inflammatory liver disease

Human intrahepatic biliary epithelial cells (HIBEC) are known to be the target of immune attack in various conditions involving the liver such as acute and chronic rejection, primary biliary cirrhosis, and primary sclerosing cholangitis. This has been attributed to their ability to express immune recognition elements, such as the DR region of the human leucocyte antigen (HLA-DR), ${ }^{1}$ which are believed to facilitate $T$ cell activation.

$\mathrm{T}$ cell activation occurs as a result of $\mathrm{T}$ cell receptor binding to an antigenic peptide held within the "groove" of MHC molecules expressed by antigen presenting cells. In addition to constitutive expression of MHC, "professional" antigen presenting cells also express cell surface costimulatory molecules, such as CD54 (ICAM-1) and members of the B7 family (CD80 and CD86), which are essential for effective $\mathrm{T}$ cell activation. Although it has been argued that any cell which expresses MHC class II can present antigen, it has now become accepted that in the absence of B7, $\mathrm{T}$ helper cells recognise antigen but do not proliferate and are rendered unresponsive to subsequent stimulation by the same antigen. ${ }^{2}$ Expression of B7 by normal human HIBEC has not been demonstrated either in vitro $^{3}$ or in vivo, ${ }^{3-6}$ although somewhat conflicting results have been reported in liver disease. ${ }^{4-6}$ Furthermore, the requirement of members of the B7 family for the activation of cytotoxic $\mathrm{T}$ cells is not as well established and there is evidence for a B7 independent pathway of activation, which may use other $\mathrm{T}$ cell accessory molecules such as CD $44 .^{78}$

CD44, the lymphocyte homing receptor, is a type I transmembrane glycoprotein. ${ }^{9}$ The principal ligand for CD44 is hyaluronan, although CD44 and its splice variants can also bind other extracellular matrix components such as collagen, fibronectin, and laminin, as well as heparin and glycosoaminoglycans. ${ }^{10}$ In the immune system, CD44 promotes $T$ cell activation and is central to the migration of memory and effector cells to sites of inflammation. The functional significance of CD44 expression by epithelial cells is less well defined, although CD44 can bind a variety of growth factors and metalloproteinases. ${ }^{10}{ }^{11}$ Thus the increased expression of CD44 on epithelial cells in various inflammatory conditions $\mathrm{s}^{12-14}$ has led to the suggestion that CD44 may play a significant role in mediating inflammation, perhaps by facilitating lymphoepithelial interactions.

We have shown previously that several immune recognition elements, including CD44, are expressed in vitro constitutively by HIBEC, but not by hepatocyte derived cell lines. ${ }^{15}$ Our aim in this study was to investigate the expression of CD44 in normal liver tissue and to determine whether this was altered in chronic liver disease, particularly primary biliary sclerosis and primary sclerosing cholangitis, where progression is caused by immune

\section{Methods}

TISSUES

Formalin fixed, paraffin embedded tissue was obtained from normal uninvolved liver from patients undergoing partial hepatectomy for mediated destruction of the bile ducts. 
Table 1 Unconjugated antibodies for immunoperoxidase labelling of normal and diseased liver tissue

\begin{tabular}{lll}
\hline Antibody* & Antigen & Antigen retrieval \\
\hline BRIC222 (1) & CD44 core epitope 1 & Microwave \\
BRIC235 (1) & CD44 core epitope 2 & Microwave \\
KZ1 (1) & CD44 core epitope 3 & Microwave \\
HERMES III (2) & CD44 core epitope 3 & Microwave \\
CR3/43 (3) & HLA-DR & Microwave \\
UCHT-1 (3) & CD3, TcR complex & Trypsin \\
IF6 (4) & CD4, T helper cells & Microwave \\
RFT8 (5) & CD8, cytotoxic T cells & Microwave \\
UCHL-1 (4) & CD45RO, memory T cells & Microwave \\
\end{tabular}

CD44 epitopes follow the designation of Anstee et al, $1991 .^{42}$ Antigen retrieval protocols were as described previously. ${ }^{16}$

^Reagent sources: (1) IBGRL Research Products, Bristol; (2) the generous gift of Dr S Jalkanen (Turku, Finland); (3) Dako Ltd (High Wycombe, Bucks); (4) Novocastra Laboratories Ltd (Newcastle Upon Tyne); (5) the generous gift of Professor G Janossy (Royal Free Hospital, London).

metastatic carcinoma (9), and transplant hepatectomy specimens from patients with primary biliary sclerosis (12) and primary sclerosing cholangitis (8). Blocks were selected which showed adequate numbers of residual bile ducts. For comparative purposes, tissues were included from the transplanted livers of patients with autoimmune hepatitis (3), hepatitis C (3), and one case of secondary sclerosing cholangitis caused by iatrogenic bile duct stricture from a cholecystectomy 10 years previously.

IMMUNOHISTOLOGY

Paraffin wax embedded sections ( $5 \mu \mathrm{m}$ thick) were dewaxed and rehydrated through graded alcohols. Endogenous peroxidase activity was blocked by $0.3 \%$ (vol/vol) $\mathrm{H}_{2} \mathrm{O}_{2}$ in methanol for 30 minutes at room temperature. To expose
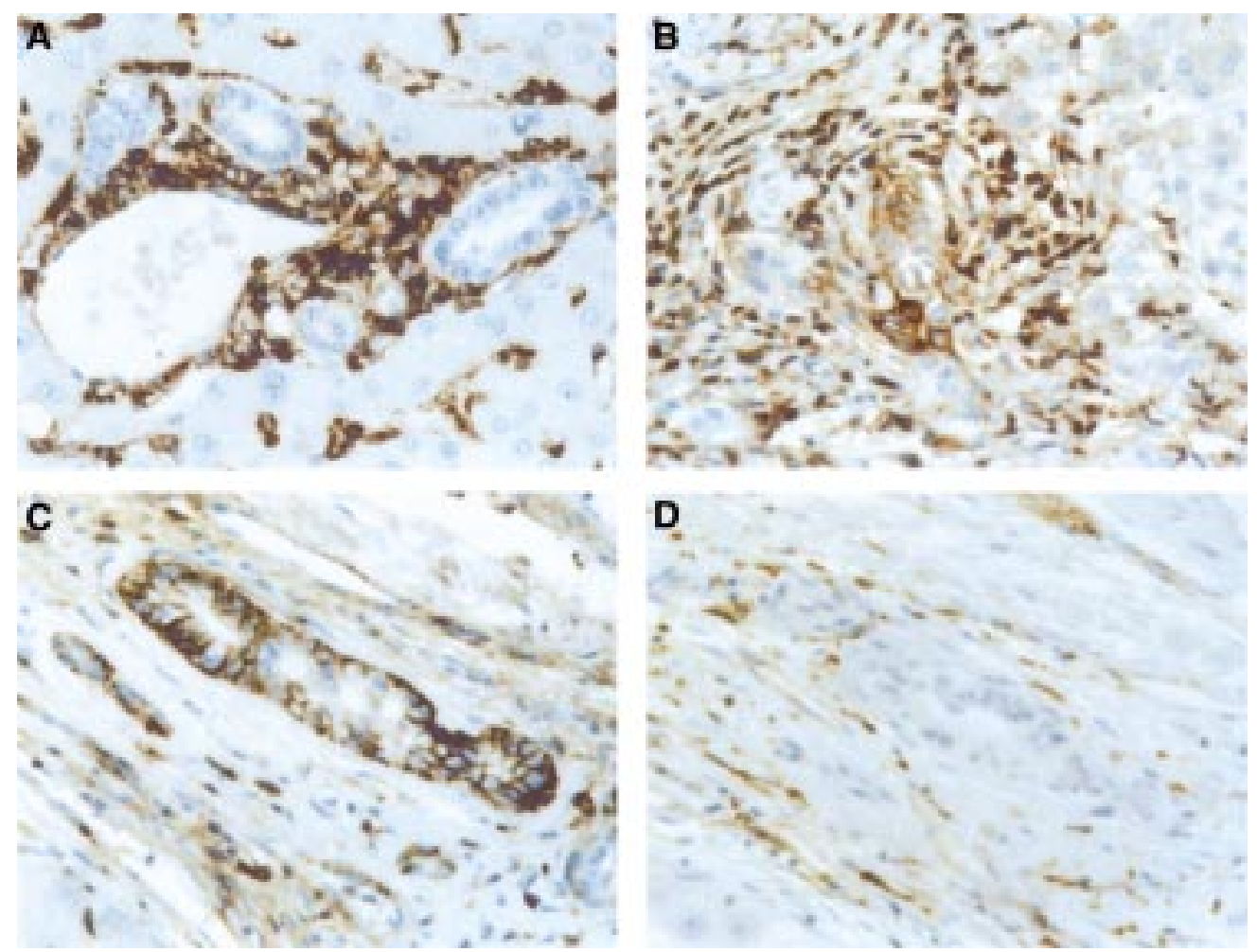

Figure 1 Expression of CD44 in normal liver (top left, $A$ ) and a liver affected with primary biliary cirrhosis (top right, $B)$ showing induction of expression on the bile ducts in this condition. Also shown is expression of CD44 (bottom left, C) and HLA-DR (bottom right, D) on the same area in primary sclerosing cholangitis, showing that the CD44 positive bile duct did not co-express HLA-DR. antigens masked by processing the tissue, sections were either boiled in $10 \mathrm{mM}$ citrate buffer, $\mathrm{pH}$ 6.0, for 10 minutes and washed (microwave retrieval), or treated with $0.05 \%$ trypsin in $0.1 \%$ calcium chloride, $\mathrm{pH} 7.8$, for 10 minutes (table 1). Non-specific secondary antibody binding and endogenous biotin were blocked by serum and avidin/biotin solutions (Vector Laboratories), respectively. Sections primary antibody for 60 minutes, biotinylated secondary antibody for 30 minutes, and streptavidin-biotin-horseradish peroxidase complex ("ABC," Dako) for 30 minutes, as described previously. ${ }^{16}$ The primary antibodies used are listed in table 1 . Antibody binding was visualised by a peroxidase activated diaminobenzidine substrate reaction. Sections were counterstained with Harris's haematoxylin, blued in Scott's tap water, dehydrated, cleared, and mounted. Normal urothelium served as the positive control tissue for CD44 core epitopes, ${ }^{17}$ and tonsil was used as the positive control for the HLA-DR and T cell antibodies. Negative controls, from which primary antibody was omitted, were included in all experiments.

\section{Results}

EXPRESSION OF CD44

In normal liver, the expression of core epitopes 1,2 , and 3 of CD44 was identical and confined to Kupffer cells, endothelial cells, and the stromal component of the portal triad. No expression of CD 44 was detected in biliary epithelial
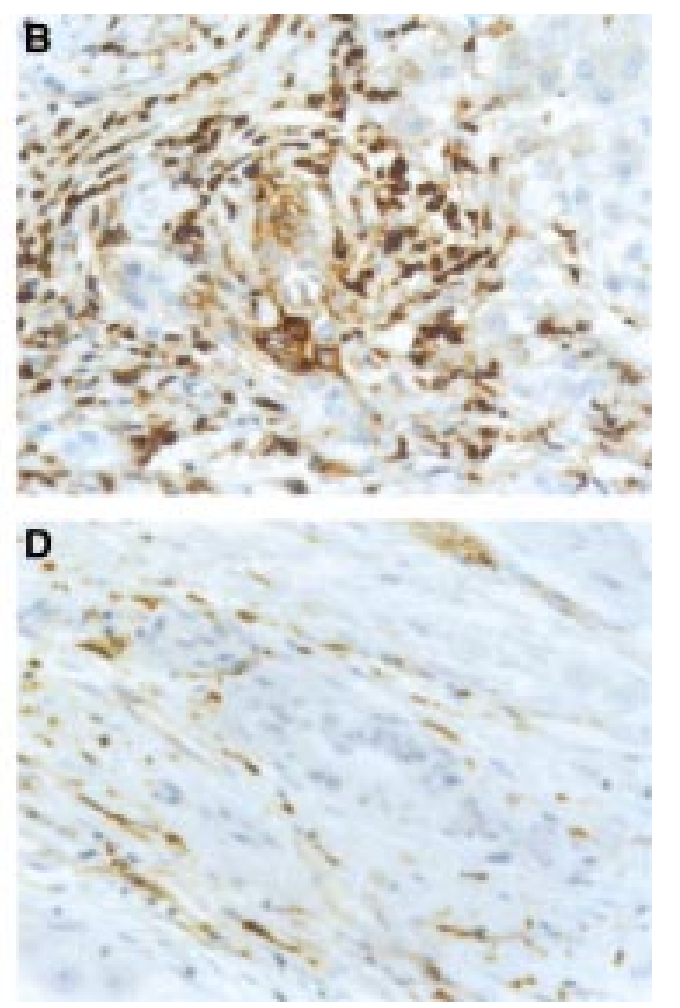
were incubated sequentially with pretitred 
Table 2 Expression of CD44 and HLA-DR on intrahepatic bile ducts

\begin{tabular}{lcc}
\hline Specimens & CD44 & HLA-DR \\
\hline Normal (n=9) & - & - \\
Primary biliary cirrhosis $(\mathrm{n}=12)$ & ++ & + \\
Primary sclerosing cholangitis $(\mathrm{n}=8)$ & ++ & + \\
Autoimmune hepatitis $(\mathrm{n}=3)$ & $+/-$ & $+/-$ \\
Hepatitis C $(\mathrm{n}=3)$ & $+/-$ & $+/-$ \\
Secondary sclerosing cholangitis $(\mathrm{n}=1)$ & ++ & + \\
\hline,$+-<5 \%$ positive ducts;,$+ 5-50 \%$ positive ducts;,$++>50 \%$ \\
positive ducts.
\end{tabular}

cells or hepatocytes in any specimen of normal liver (fig 1).

Sections from all 27 transplant hepatectomy specimens showed CD44 expression in bile duct epithelial cells. The proportion of ducts affected was much higher in biliary disease than in chronic hepatitis (table 2). By contrast, no CD44 expression was detected in hepatocytes from any of the diseased livers.

Most (65-90\%) of the large, intermediate, and septal bile ducts from patients with primary biliary sclerosis and primary sclerosing cholangitis showed basolateral expression of CD44 core epitopes 1 and 2 (fig 1; table 2). Basolateral expression of CD44 core epitopes 1 and 2 was also observed in $25-50 \%$ of the smaller ducts. In the six cases of chronic hepatitis, less than $5 \%$ of the ducts were CD 44 positive, with positivity seen least often in hepatitis C (table 2). CD44 core epitope 3 was not detected in bile ducts by either of the two different anti-CD 44 core 3 antibodies (Hermes 3 and $\mathrm{KZ1}$ ).

RESIDENT LYMPHOCYTES

In addition to the expression of CD44 in the bile ducts of the diseased tissues, lymphocytes throughout the tissues (normal and diseased) were positive for CD44 core epitopes 1 and 2 . However, the majority (90\%) of T cells did not express detectable CD44 core epitope 3. Virtually all $(\geqslant 98 \%)$ of the $\mathrm{T}$ cells also expressed CD45RO. Thus $\mathrm{T}$ cells within the diseased livers were predominantly of a CD45RO ${ }^{\text {hi }}, \mathrm{CD}_{4} 4^{\text {hi }}$ "memory" phenotype. Expression of CD44 on T cells was particularly evident in germinal centres seen at the edges of damaged ducts. It was also observed that cells in the pre-B cell region of the germinal centres did not express CD44. T cell expression of CD44 was also seen in autoimmune hepatitis and hepatitis $\mathrm{C}$.

In normal liver, $\mathrm{CD}^{+} \mathrm{T}$ cells were the predominant $T$ cell population, with a $2: 1$ ratio of $\mathrm{CD}^{+}$to $\mathrm{CD}^{+}$. The $\mathrm{CD}^{+} \mathrm{T}$ cells in normal liver were located between hepatocytes and in the stroma of the portal tracts, with occasional intraepithelial location in septal ducts. By contrast, in diseased livers the majority of $\mathrm{T}$ cells (60-70\%) were $\mathrm{CD} 4^{+}$lymphocytes. There was a heavy infiltrate of predominantly $\mathrm{CD}^{+} \mathrm{T}$ cells in the bile ducts in primary biliary sclerosis and primary sclerosing cholangitis, although CD4 cells were often observed just beneath the basement membrane of bile ducts. Many of the lymphocytes in the diseased tissues expressed HLA-DR, which was particularly evident where there was a germinal centre.
EXPRESSION OF HLA-DR

In normal liver, HLA-DR expression was restricted to Kupffer cells (high expression) and stromal cells of the portal tracts (low expression). In primary biliary sclerosis and primary sclerosing cholangitis, $30-50 \%$ of the large intermediate and septal bile ducts and approximately $25 \%$ of the smaller bile ducts expressed HLA-DR (table 2). Hepatocytes did not express HLA-DR in either primary biliary sclerosis or primary sclerosing cholangitis. Overall, there were a greater number of Kupffer cells in the diseased livers, all of which expressed HLA-DR (fig 1). In some of the diseased tissues, HLA-DR expression was observed on endothelial cells within the portal tracts, although in general endothelial cells did not express HLA-DR. Expression of HLA-DR was high on many of the hepatocytes in the samples of hepatitis $C$ and autoimmune hepatitis, but HLA-DR expression was apparent only in a small percentage of ducts (less than $5 \%$; table 2). HLA-DR positive bile ducts coexpressed CD44 although not all CD44 positive ducts expressed HLA-DR (fig 1).

\section{Discussion}

Our data suggest that CD44 expression by HIBEC is virtually a universal feature of inflammatory liver disease. Furthermore, expression of CD44 appeared to correlate with bile duct damage, as the proportion of positive ducts was higher in primary biliary sclerosis and primary sclerosing cholangitis than in autoimmune hepatitis or hepatitis C. CD44 expression was observed even in the one case of iatrogenic secondary sclerosing cholangitis. These observations suggest that CD44 expression is a function of biliary cell response to stress or damage. Although no CD44 expression was observed by HIBEC in normal liver, our previous studies have shown that cultured HIBEC express CD44 in the absence of any apparent exogenous inducing agent. ${ }^{15}$ Such observations suggest that expression of CD44 by HIBEC may be associated with cell proliferation. A connection between epithelial cell proliferation and expression of CD44 has been suggested previously, ${ }^{18}$ although subsequent studies have contradicted this view. ${ }^{19} 20$ Thus there are likely to be additional mechanisms involved in the induction of CD44 expression. We have previously shown that interleukin (IL) -4 and IL-13 can upregulate CD44 and CD44 splice variant 6 (CD44v6) expression in cultured colonocytes. ${ }^{21}$ However, neither IL-4 nor the cytokines interferon (IFN) $\gamma$, tumour necrosis factor $\alpha$, transforming growth factor $\beta$, IL- 6 , and IFN $\alpha$ had any apparent effect on CD44 expression in cultured HIBEC. ${ }^{15}$ Expression of CD44 has been induced on keratinocytes following ligation of CD40 by its ligand CD40L (CD154). ${ }^{22}{ }^{23}$ As we have found that HIBEC express CD40 constitutively $^{15}$ and expression of CD40L is confined to activated $\mathrm{T}$ cells, such findings suggest that there may be a direct involvement of the infiltrating $\mathrm{T}$ cells in the induction of CD44 in HIBEC. This may be why CD44 
upregulation is associated with inflammatory processes in other epithelial organs. ${ }^{12-14}$

In primary biliary sclerosis and primary sclerosing cholangitis, expression of CD44 core epitopes 1 and 2 was detected on the majority of bile ducts. The lack of expression of core epitope 3 on HIBEC on these sections probably reflects an epitope masking phenomenon, as HIBEC have been shown to express CD44 core epitope 3 in vitro. ${ }^{15}$ Similar differential detection of core epitopes 1 and 2 was found on a large majority of $\mathrm{T}$ lymphocytes. Although no specific functional properties have been ascribed to the CD44 core epitopes recognised by the various antiCD44 antibodies, we have previously described the masking of core epitope 3 in intestinal epithelium, ${ }^{24}$ a finding suggestive of CD44 receptor occupancy in situ. The observation that almost all $\mathrm{T}$ cells in the liver were high expressors of CD44 is consistent with their CD45R0 "hi "memory cell" phenotype, ${ }^{25}$ and agrees with a previous report. ${ }^{26}$ Although not the primary purpose of this study, the finding that many $T$ cells also expressed HLA-DR in inflamed livers is evidence that these cells were activated.

One function for CD44 in epithelial cells appears to be the sequestration of chemokines, ${ }^{27} 28$ cytokines, and growth factors. ${ }^{10}{ }^{11}$ In the diseased liver, presentation of chemokines could be a mechanism promoting chemotaxis of lymphocytes and other inflammatory cells to the bile ducts. CD 44 also binds the Eta-1/osteopontin protein ${ }^{29}$ thought to be an important regulator of immune function. ${ }^{30} 31$ CD44 has been shown to bind hepatocyte growth factor/scatter factor, ${ }^{32}$ a potent mitogen for hepatocytes and biliary epithelial cells ${ }^{33}$ which is thought to play a key role in liver regeneration..$^{34} \mathrm{CD} 44$ can also bind heparinbinding epidermal growth factor and fibroblast growth factor (FGF), ${ }^{36}{ }^{37}$ both of which can be produced by $\mathrm{T}$ cells. ${ }^{38}$ In addition to its potent role as a mitogen for mesenchymal cells, both FGF and hepatocyte growth factor have been shown to promote restitution during wound healing by intestinal epithelial cells, ${ }^{39}{ }^{40}$ and may also promote secretion of IFN $\gamma$ by natural killer (NK) cells, ${ }^{41}$ which would serve to upregulate HLA-DR expression.

Although primary biliary sclerosis and primary sclerosing cholangitis have quite different histological manifestations, the expression patterns of HLA-DR and CD44 were strikingly similar. Furthermore, the distribution and phenotypes of the infiltrating lymphocytes were also very similar. This suggests some commonality of immunological mechanisms in the two diseases. The finding that CD44 and HLA-DR were generally induced on the larger interlobular ducts in primary biliary sclerosis reflects the fact that a significant proportion of the cases included in this study were of end stage disease in which there was a marked reduction in numbers of small ducts. Our finding that HLA-DR was not expressed by the majority of the bile ducts in the diseased livers concurs with a recently published study. ${ }^{5}$ Expression of HLA-DR reflects the presence of IFN $\gamma$, of which activated proinflammatory $\mathrm{T}$ cells and NK cells are the only known sources. This would suggest that HLA-DR expression by HIBEC is not a primary event in the development of primary biliary sclerosis and primary sclerosing cholangitis. Thus HLA-DR may have a role in the development rather than initiation of chronic progressive biliary diseases.

\section{CONCLUSIONS}

We suggest that the induction of CD44 on many of the bile ducts in primary biliary sclerosis and primary sclerosing cholangitis may play a primary role in the progression of disease. Although there is no direct evidence that expression of CD44 by biliary cells can directly promote $\mathrm{T}$ cell activation, its ability to bind chemokines and growth factors suggests that it plays a central role in mediating local inflammatory responses. Furthermore, we hypothesise that CD44 acts by both promoting the activation and migration of $\mathrm{T}$ cells to the bile ducts and facilitating subsequent lymphoepithelial interactions, perhaps through a B7 independent mechanism.

This work was supported by the Leeds Liver Trust. We are grateful to Mrs Lynne Hill and Mrs Christine Gascoigne for excellent technical assistance and to Professor M S Losowsky for discussions and support.

1 Ayres RC, Neuberger JM, Shaw J, et al. Intercellular adhesion molecule-1 and MHC antigens on human intrahepatic bile duct cells: effect of pro-inflammatory cytokines. Gut 1993;34:1245-9.

2 Lombardi G, Hargreaves R, Sidhu S, et al. Antigen presentation by $T$ cells inhibits IL-2 production and induces IL-4 release due to altered cognate signals. F Immunol 1996;156: 2769-75.

3 Leon MP, Kirby JA, Gibbs P, et al. Immunogenicity of biliary epithelial cells: study of the expression of B7 molecules. F Hepatol 1995;22:591-5.

4 Tsuneyama K, Van de Water J, Leung PSC, et al. Abnormal expression of the E2 component of the pyruvate dehydrogenase complex on the luminal surface of biliary epithelium occurs before major histocompatibility complex class II and BB1/B7 expression. Hepatology 1995;21:1031-7.

5 Spengler U, Leifeld L, Braunschweiger I, et al. Anomalous expression of costimulatory molecules B7-1, B7-2 and CD28 in primary biliary cirrhosis. F Hepatol 1997;26:31-6.

6 Tsuneyama K, Harada K, Yasoshima M, et al. Expression of co-stimulatory factor B7-2 on the intrahepatic bile ducts in primary biliary cirrhosis and primary sclerosing cholangitis: an immunohistochemical study. $\mathcal{F}$ Pathol 1998; 186: $126-30$.

7 Conrad P, Rothman BL, Kelley K, et al. Mechanism of peripheral $\mathrm{T}$ cell activation by coengagement of CD 44 and CD2. F Immunol 1992; 149:1833-9.

8 Funaro A, Spagnoli GC, Momo M, et al. Stimulation of T cells via CD44 requires leukocyte-function-associated antigen interactions and interleukin-2 production. Нит Iттиnol 1994;40:267-8.

9 Stamenkovic I, Amiot M, Pesando JM, et al. A lymphocyte molecule implicated in lymph node homing is a member of the cartilage link protein family. Cell 1989;56:1057-62.

10 Naot D, Sionov RV, Ish-Shalom D. CD44: structure, function, and association with the malignant process. Adv Cancer Res 1997;71:241-319.

11 Sneath RJS, Mangham DC. The normal structure and function of CD 44 and its role in neoplasia. 7 Clin Pathol function of CD44 and its role

12 Fan XG, Kelleher D, Fan XJ, et al. Helicobacter pylori increases proliferation of gastric epithelial cells. Gut 1996;38:19-22.

13 Holgate ST. Asthma: a dynamic disease of inflammation and repair. Ciba Foundation Symp 1997;206:5-28.

14 Zhu SN, Nolle B, Duncker G. Expression of adhesion molecule CD44 on human corneas. Br f Ophthalmol 1997;81: $80-4$

15 Cruickshank SM, Southgate J, Selby PJ, et al. Expression and cytokine regulation of immune recognition elements by normal human biliary epithelial and established liver cell lines in vitro. F Hepatol 1998;29:550-8.

16 Southgate J, Trejdosiewicz LK. Immunolabelling of cells and tissues: approaches and pitfalls. Hum Reprod 1997; 12(suppl): $65-75$.

17 Southgate J, Trejdosiewicz LK, Smith B, et al. Patterns of splice variant CD44 expression by normal human urothelium in situ and in vitro and by bladder-carcinoma cell lines. Int $\mathcal{f}$ Cancer 1995;62:449-56. 
18 Abbasi AM, Chester KA, Talbot IC, et al. CD44 is associated with proliferation in normal and neoplastic 1995-2002.

19 Takahashi K, Stamenkovic I, Cutler M, et al. CD44 hyaluronate binding influences growth kinetics and tumorigenicity of human colon carcinomas. Oncogene 1995;11 2223-32.

20 Furuta K, Zahurak M, Yang XL, et al. Relationship between CD44 expression and cell proliferation in epithelium and stroma of colorectal neoplasms. Am f Pathol 1996;149: $1147-55$

21 Trejdosiewicz LK, Morton R, Yang Y, et al. Interleukins 4 and 13 upregulate expression of CD44 in human colonic epithelial cell lines. Cytokine 1998;10:756-65.

22 Shinde S, Wu Y, Guo Y, et al. CD40L is important for induction of, but not response to, costimulatory activityICAM-1 as the second costimulatory molecule rapidly up-regulated by CD40L. F Immunol 1996;157:2764-8.

23 Guo Y, W Y Y, Shinde S, et al. Identification of a costimulatory molecule rapidly induced by $\mathrm{CD} 40 \mathrm{~L}$ as $\mathrm{CD} 44 \mathrm{H}$. $\mathcal{F}$ Exp Med 1996;184:955-61.

24 Sillett HK, Southgate J, Howdle PD, et al. Expression of activation and costimulatory elements by human intestina intraepithelial lymphocytes. Scand F Immunol 1999;50:5260.

25 Camp RL, Kraus TA, Birkeland ML, et al. High levels of CD44 distinguish virgin from antigen-primed B cells. f Exp Med 1991;173:763-6.

26 Leon MP, Spickett G, Jones DE, et al. CD4+ T cell subsets defined by isoforms of CD45 in primary biliary cirrhosis. Clin Exp Immunol 1995;99:233-9.

27 Tanaka Y, Adams D, Hubscher S, et al. T cell adhesion induced by proteoglycan-immobilised cytokine MIP-1b. Nature 1993;361:79-82

28 Jackson DG, Bell JI, Dickinson R, et al. Proteoglycan forms of the lymphocyte homing receptor CD44 are alternatively spliced variants containing the v3 exon. $f$ Cell Biol 1995;128:673-85.

29 Weber GF, Ashkar S, Glimcher MJ, et al. Receptor-ligand interaction between CD44 and osteopontin (Eta-1). Science 1996;271:509-12

30 Weber GF, Cantor H. The immunology of Eta-1/ osteopontin. Cytokine Growth Factor Rev 1996;7:241-8.
31 Uede T, Katagiri Y, Iizuka J, et al. Osteopontin, a coordinator of host defense system: a cytokine or an extracellular adhesive protein? Microbiol Immunol 1997;41:641-8.

32 Pals ST, Taher TE, Van der Voort R, et al. Regulation of adhesion and migration in the germinal center microenvironment. Cell Adhes Commun 1998;6:111-16.

33 Joplin R, Hishida $\mathrm{T}$, Tsubouchi $\mathrm{H}$, et al. Human intra-hepatic biliary cells proliferate in response to human hepatocyte growth factor. F Clin Invest 1992;90:1284-89.

34 LaBrecque D. Liver regeneration: a picture emerges from the puzzle. Am f Gastroenterol 1994;89(suppl):S86-96.

35 Fausto N, Laird A, Webber E. Liver regeneration 2-role of growth factors and cytokines in hepatic regeneration. FASEB f 1995;9:1527-36.

36 Hardingham TE, Fosang AJ. Proteoglycans: many forms and many functions. FASEB 7 1992;6:861-70.

37 Bennett KL, Jackson DG, Simon JC, et al. CD44 isoforms containing exon V3 are responsible for the presentation of heparin-binding growth factor. F Cell Biol 1995;128:68798.

38 Blotnick S, Peoples GE, Freeman MR, et al. T lymphocytes synthesize and export heparin-binding epidermal growth factor-like growth factor and basic fibroblast growth factor, mitogens for vascular cells and fibroblasts: differential production and release by CD4+ and CD8+ T cells. Proc Natl Acad Sci USA 1994;91:2890-4.

39 Dignass AU, Tsunekawa S, Podolsky DK. Fibroblast growth factors modulate intestinal epithelial cell growth and migration. Gastroenterology 1994;106:1254-62.

40 Dignass AU, Lynch-Devaney K, Podolsky DK. Hepatocyte growth factor/scatter factor modulates intestinal epithelial cell proliferation and migration. Biochem Biophys Res Com 1994;202:701-9.

41 Lewis CE, Ramshaw AL, Lorenzen J, et al. Basic fibroblast growth factor and interleukins 4 and 6 stimulate the release of IFN-gamma by individual NK cells. Cell Immunol 1991; 132:158-67.

42 Anstee DJ, Gardner B, Spring FA, et al. New monoclonal antibodies in CD44 and CD58: their use to quantify CD44 and CD58 on normal human erythrocytes and to compare the distribution of CD44 and CD58 in human tissues. Immunology 1991;74:197-205. 